\title{
Scanning electron microscopy of superficial defects in articular cartilage
}

\author{
F. N. GHADIALLY, R. L. AILSBY, AND A. F. ORYSCHAK \\ From the Department of Pathology, University Hospital, University of Saskatchewan, Saskatoon, Canada
}

It has long been recognized that the power of articular cartilage to regenerate and repair wounds in its substance is feeble or nonexistent (Hunter, 1743; Paget, 1853). This is largely due to the fact that chondrocytes in the mature animal are virtually incapable of mitotic division (Elliot, 1936; Mankin, 1962a,b, 1963). It is now believed that superficial injuries (partial-thickness defects) which do not violate subchondral bone do not heal, but deep injuries (full-thickness defects) which penetrate subchondral bone heal by repair tissues arising from marrow spaces (Bennett and Bauer, 1932, 1935; Mankin, 1962b; De Palma, McKeever, and Subin, 1966; Campbell, 1969; Ghadially, Fuller, and Kirkaldy-Willis, 1971; Fuller and Ghadially, 1972).

In a recent ultrastructural study of partial-thickness defects in the articular cartilage of immature rabbits, we (Fuller and Ghadially, 1972) confirmed the fact that articular cartilage fails to generate a significant repair reaction capable of filling the defect; but we also showed that some attempt at repair is made, for the surviving chondrocytes show evidence of heightened metabolic activity and by about 6 months there is a remodelling of the surface of the defect which becomes covered by a layer of new matrix containing fine collagen fibres deployed tangentially to the articular surface.

It is thought that the size of both deep and superficial defects is probably slightly reduced by a phenomenon referred to as matrix flow, whereby it is envisaged that pressure exerted by weight bearing 'rounds off' the edges of the defect and also makes the matrix flow into the 'hollow' of the defect. Though the existence of such a phenomenon has been recognized (DePalma and others, 1966; Campbell, 1969; Ghadially and others, 1971; Fuller and Ghadially, 1972), we and others have considered it to be of minor importance.

Recently we examined the surface of superficially injured cartilage with the scanning electron microscope and were surprised to find that matrix flow is no trivial phenomenon, but one which produces hitherto unsuspected changes on the injured surface worthy of careful study and deliberation. We also feel that the term 'matrix flow' should be replaced by 'cartilage flow', for there is little reason to believe that it is only the matrix which flows and that the cells are left behind. The purpose of this paper is to compare the scanning electronmicroscopical appearance of freshly injured cartilage with that collected 6 months after injury and to correlate the changes seen with previous light and electron microscopical studies executed by us and others.

\section{Materials and methods}

\section{EXPERIMENTAL PROCEDURES}

Eighteen rabbits of both sexes, between 3 and 4 months old, were used for this experiment. Under strict aseptic precautions and Nembutal anaesthesia the right knee joint was opened. A thin shaving of articular cartilage approximately $2 \mathrm{~mm}$ in diameter was removed tangential to the surface with the aid of a scalpel from the weight bearing area of the medial femoral condyle. Care was taken to see that in no instance did the scalpel contact calcified tissue.

The shaving of cartilage obtained from each joint was collected in $10 \%$ formalin for study with the light microscope. In some cases serial sections prepared from paraffin-embedded material were studied, in others the entire piece of cartilage was stained and mounted, and its deep surface examined to ensure that calcified tissue was not being accidently removed by our technique.

Six animals were killed immediately after operation, the remaining 12 were killed 6 months later. The lower end of the femur was freed from the muscle and joint capsule and then collected for scanning electron microscopy.

\section{PROCESSING OF TISSUES FOR SCANNING}

ELECTRON MICROSCOPY

The articular surface of the lower end of the femur was rinsed thoroughly in four changes of normal saline to remove synovial fluid and any blood that might have contaminated the articular surface during collection of the specimen. The specimen was then fixed in $2 \%$ glutaraldehyde in cacodylate buffer for one to two weeks and dehydrated in increasing concentrations of ethanol over the course of another week. A portion of the medial femoral condyle bearing the superficial defect was then dissected off with a fine fret saw, rinsed in alcohol, and mounted on a microscope stub with electrodag. Specimens were vacuum coated with gold and examined in a Cambridge 'stereoscan' electron microscope. 


\section{Results}

FRESHLY MADE SUPERFICIAL INJURY

The removal of a thin shaving of cartilage produces a circular or oval defect on the articular surface. The appearance of such an injury as seen with the scanning electron microscope is illustrated in Figs 1 and 2. The margin of the defect is quite sharp as evidenced

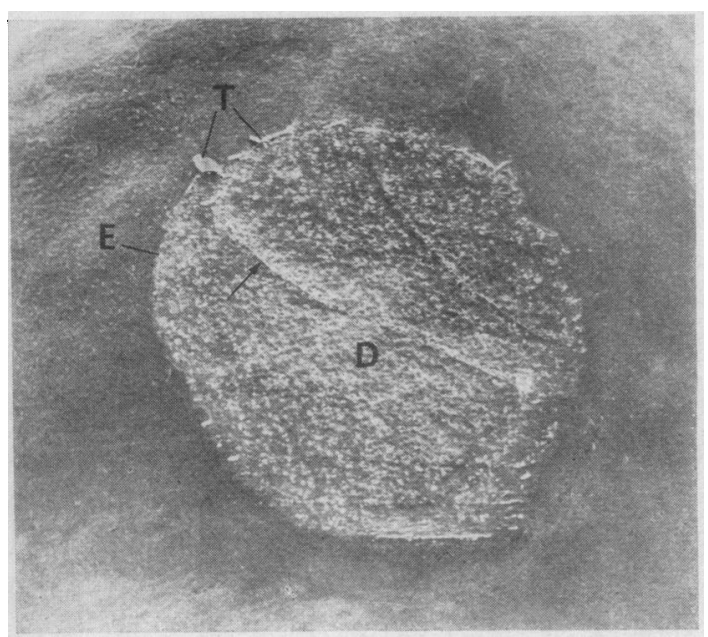

FIG. 1 A low-power view of the medial femoral condyle showing a partial-thickness defect $(D)$. The edge $(E)$ of the defect is sharply demarcated from surrounding cartilage. Note the cutting marks (arrow) and the few small tags of tissue $(T)$ raised by the knife. Specimen collected a few minutes after surgery. $\times 20$ by the abrupt transition between the area of the defect and the surrounding cartilage. The surface of the defect shows linear marks attributed to the unsteadiness of the human hand and slight imperfections of the cutting edge employed to produce these defects. The shaving off of a thin layer of cartilage lays bare many chondrocytes and their lacunae. Previous light and electron microscopical studies (Fuller and Ghadially, 1972) on similar defects produced by us has shown that the deepest part of these defects (i.e. near the centre of defect) lies in the depths of zone 2 or within the superficial parts of zone 3 . The appearances seen with the scanning microscope in the present study were consistent with this observation, but the exact depth of these could not be ascertained by this technique. Light microscopical studies of the excised fragment of cartilage, however, confirmed that the injuries reported in the present study are comparable to those reported by us previously (Fuller and Ghadially, 1972).

\section{SIX-MONTH-OLD DEFECTS}

The appearances seen in all 12 defects collected at this time interval were essentially similar. The margin of the defect was no longer sharply demarcated from the surrounding cartilage. In places the junction between these two zones could still be discerned, but often this line of demarcation was difficult to determine (Fig. 3). The floor or surface of the defect was traversed by sheets, bands, ribbons, and rope-like and thread-like structures which seemed to arise from the surface and margin of the defect (Figs 3 to 5). Collectively they may be referred to as 'flow forma-

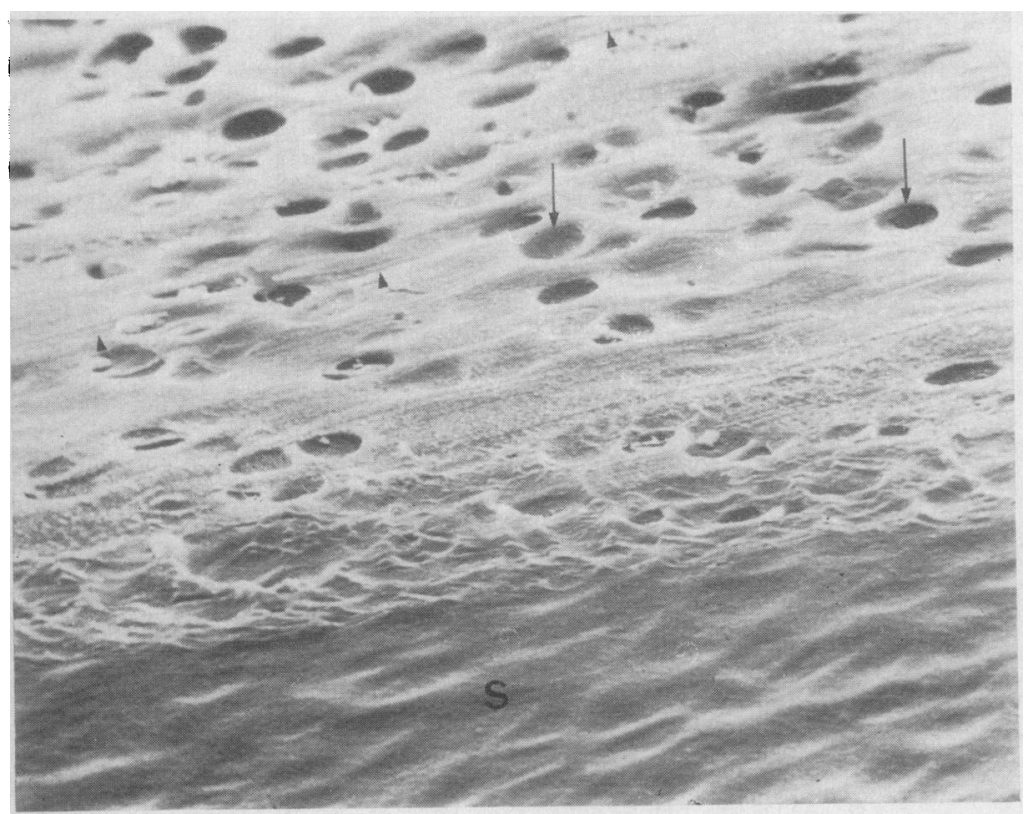

FIG. 2 Edge of a defect on medial femoral condyle collected a few minutes after surgery. The surface of the surrounding normal cartilage $(S)$ show's numerous shallow depressions. The surface of the defect shows numerous chondrocyte lacunae (arrows) laid bare by the knife. Note also the fine score lines made by the knife (arrowheads). $\times 400$ 
tions', for these structures appear to be derived by a streaming of the cartilage substance. Wave-like formations (reminiscent of an incoming tide from a calm sea onto a sandy beach) giving a vivid impression of 'flow' were frequently encountered (Fig. 6).
Though a majority of these flow formations stemmed from the margin and floor of the injured area, at times a few rope-like and thread-like formations were also seen arising from the surrounding cartilage adjacent to the defect (Figs 3 and 4).

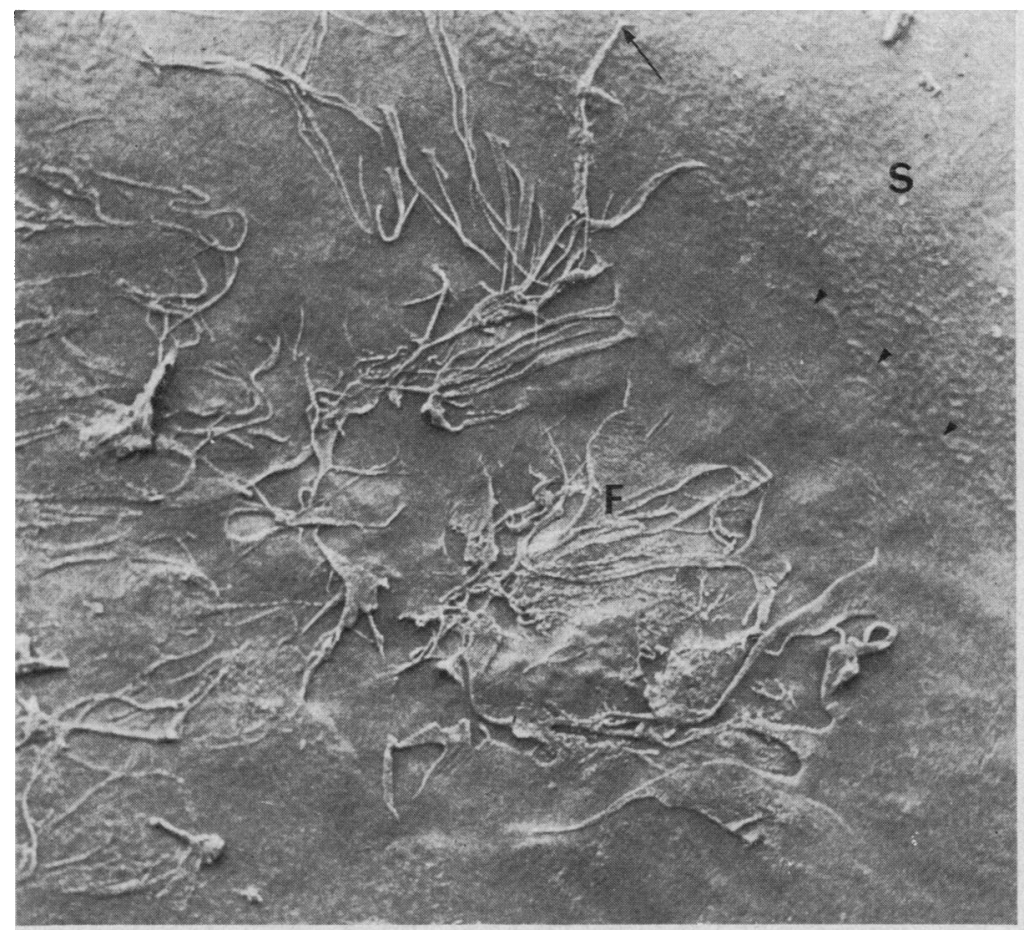

FIG. 3 Surface of a partial-thickness defect 6 months after surgery. The junction between defect and surrounding cartilage $(S)$ is clearly seen in one area (arrowheads), but the edge is rounded and indistinct over the rest of its circumference. Numerous flow formations (F) arising from the surface of the defect and one or two (arrow) arising from surrounding cartilage meander over the surface of the defect. Note how the smooth floor of the defect contrasts with the surrounding cartilage surface covered by shallow depressions. $\times 72$

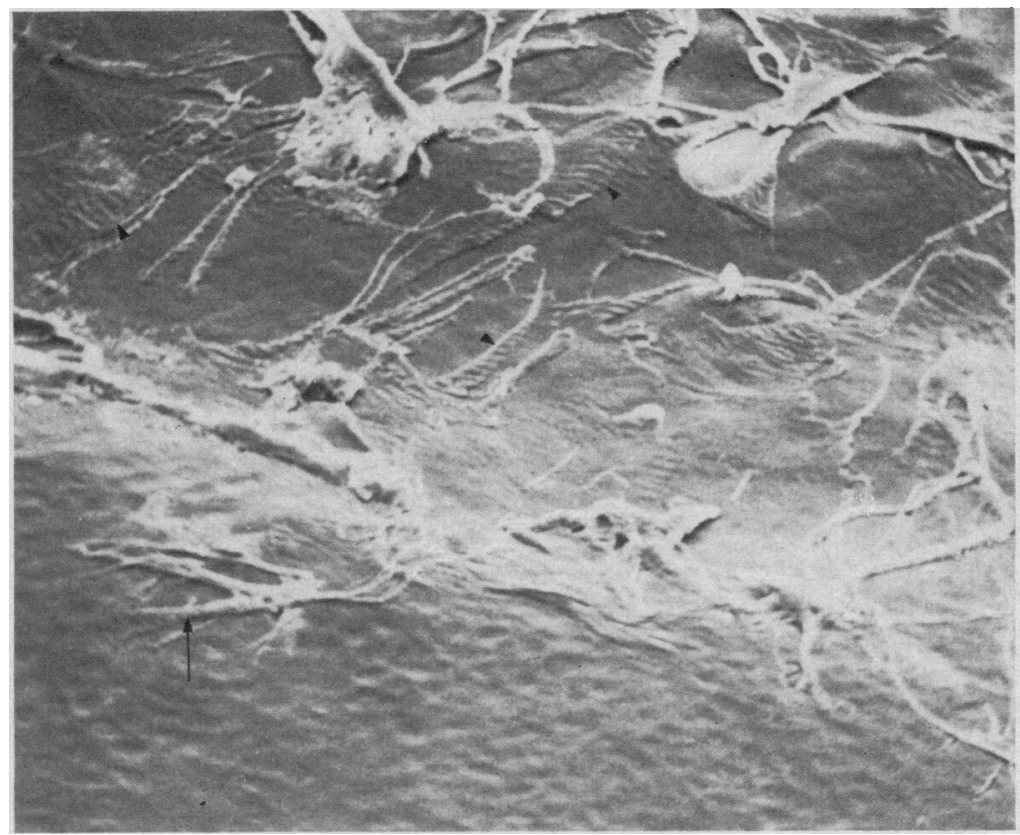

FIG. 4 Edge of a partial-thickness defect 6 months after surgery. The corrugated (arrowheads) nature of the flow formation is clearly depicted here. Though most of the flow formation arises from the surface and margin of the defect, a few (arrow) are seen springing from the surrounding cartilage. $\times 200$ 


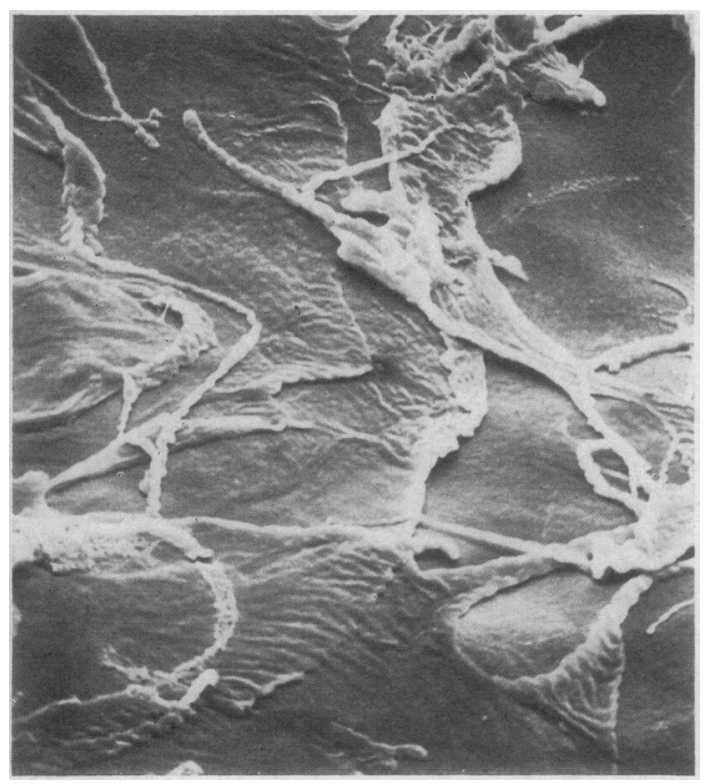

FIG.6 Wave-like formation on the floor of a partial-thickness defect, giving a vivid impression of cartilage flow. $\times 350$
The flow formations often had a corrugated, wavey, or striated appearance (Figs 4 and 5). As a rule they did not show 'pits' or 'holes' suggesting the presence of chondrocytes and lacunae in their substance. However, at times our suspicion was aroused particularly in the case of larger irregular-shaped masses associated with these flow formations, for these invariably showed many 'pits' or 'holes' in their substance (Fig. 7).

The surface of the defect was often criss-crossed by the various types of flow formation described above, but between these peered the true surface or floor of the defect which had a homogeneous fine textured appearance. This surface did not show the shallow pitted or undulating appearance now known to be characteristic of normal cartilage viewed with the scanning electron microscope (Clarke, 1971a,b). Further, we could find neither remnants of exposed chondrocyte lacunae nor the knife marks left behind when the superficial injury was made.

\section{Discussion}

The illustrations in this paper show that cartilage flow is no trivial phenomenon which merely produces a rounding off of the edge of the superficial defects 


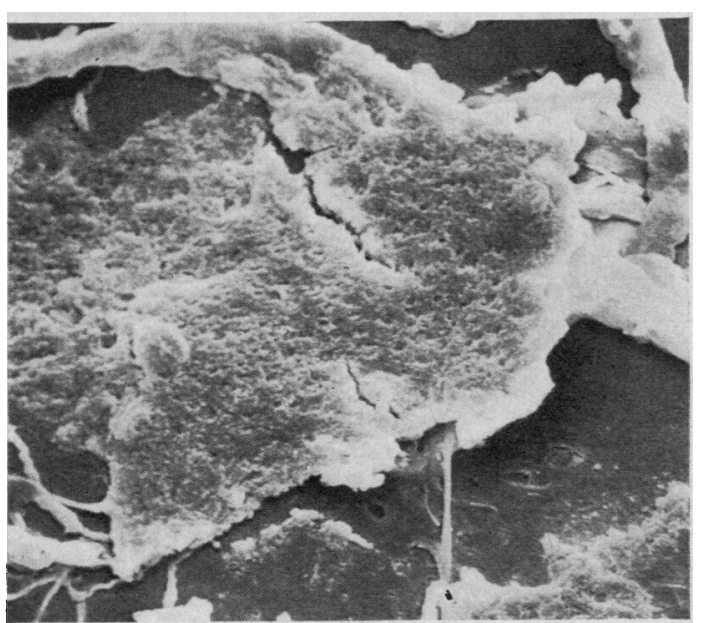

FIG. 7 High-power view of a rough-textured formation shown in Fig. 5. Note numerous pits on its surface. $\times 475$

(as had been thought in the past), but is one which produced remarkable alterations of the injured surface. The appearances seen seem to be explicable on the basis of pressure deformation (load bearing) of a plastic tissue and also perhaps friction and rubbing of the apposed articular surfaces in the region of the defect.

A widely held concept of joint lubrication is that the opposing cartilage surfaces are prevented from contacting primarily by trapped pools of synovial fluid (Walker, Sikorski, Dowson, Longfield, Wright, and Buckley, 1969). Further, it is envisaged that such fluid compressed between the cartilage surfaces forms a protective gel. The combination of these two phenomena has been called 'boosted lubrication' (Walker, Dowson, Longfield, and Wright, 1968).

It seems to us that for the operation of this lubricating mechanism an intact cartilage surface is essential, and that if the fluid film, or gel, is disrupted by a superficial defect then contact and rubbing of opposing articular surfaces is likely to ensue in the region of the defect.

In the early stages probably only the margin of the defect contacts the opposite surfaces, and this leads to a rounding off of the margins and also starts cartilage flow from this region. It is conceivable that later the entire surface of the defect may contact the opposite joint surface and further new flow formations and alterations of pre-existing formations may ensue.

It seems to us, therefore, that the pressure of loadbearing and the shearing forces that come into play as a result of joint movement are responsible for the flowing and streaming of cartilage which occurs. It is not difficult to visualize how this would produce wavelike and sheet-like formations which, as they become attenuated by pressure and movement, break up into ribbons and threads, as does the edge of a lump of pastry dough rolled by a novice. Such a mechanism may also explain how twisted rope-like structures arise from the tips and margins of the bands and ribbons (Fig. 5). It is also conceivable that shortening of collagen fibres contained in these formations (either in vivo or during preparation of the specimen) may be a factor responsible for the contorted appearance of these rope-like formations and the corrugated appearance of the sheets and the bands of flowing cartilage.

The nature of the rope-like and thread-like formations seen on the main surface of the defect is difficult to interpret. It could be that such formations are detached from the flowing sheets and ribbons and subsequently fuse to the surface of the defect, or that such formations are formed in situ. In the latter instance one would have to postulate that with time the defect becomes shallower and parts of its irregular surface make contact with the opposite joint surface and suffer shearing and rolling forces.

The fine textured floor of the defect over which the flowing ribbons and ropes meander probably represents the newly formed layer of matrix containing fine fibres which, as we have noted, forms over the injured surface by about 6 months (Fuller and Ghadially, 1972). This new zone is devoid of chondrocytes, hence it does not present shallow depressions on its surface as does the surrounding articular cartilage.

Much remains to be learned about the significance of these new flow formations revealed by the scanning electron microscope. Clearly this is not the same phenomenon as the cracking and flaking of osteoarthritic cartilage surface described by us (Meachim, Ghadially, and Collins, 1965) and others (Collins, 1949; Redler and Zimny, 1970) as fibrillation; though in retrospect it does seem that some flowing and rolling of the tags produced on the surface of fibrillated cartilage may well occur. A question that arises is whether such flow formations should be regarded as a factor which assists in filling the defect. However, this does not seem to be a very effective mechanism, for such flow formations are probably rubbed off and destroyed by joint movement and load bearing.

It would be interesting to know what would happen if load bearing and joint movement were restricted or eliminated. Our preliminary observations suggest many other points worth investigating. Clearly, further studies at earlier and later time intervals are needed and it would also be interesting to see whether the opposite joint surface is affected. Such problems are eminently suitable for study with the scanning electron microscope.

\section{Summary}

The remodelling of the surface of partial-thickness defects in the articular cartilage of immature rabbits 
was studied with the scanning electron microscope. At the end of 6 months the floor of the defect was covered by a homogeneous fine textured material which obliterated the chondrocyte lacunae exposed when the defect was made. Over the floor of the defect lay numerous sheets, bands, and rope-like and thread-like formations (flow formations) believed to be produced as a result of cartilage flow engendered by load bearing and joint movement.

This study was supported by grants from the Medical Research Council of Canada and the Canadian Arthritis and Rheumatism Society.

\section{References}

Bennett, G. A., AND Bauer, W. (1932) Amer. J. Path., 8, 499 (A study of the repair of articular cartilage and the reaction of normal joints of adult dogs to surgically created defects of articular cartilage, 'joint mice' and patellar displacement)

_ , (1935) J. Bone Jt Surg., 17, 141 (Further studies concerning the repair of articular cartilage in dog joints)

CAmpbell, C. J. (1969) Clin. Orthop., 64, 45 (The healing of cartilage defects)

Clarke, I. C. (1971a) Ann. rheum. Dis., 30, 15 (Human articular surface contours and related surface depression frequency studies)

- (1971b) J. Anat., 108, 23 (Surface characteristics of human articular cartilage-a scanning electron microscopic study)

Collins, D. H. (1949) In 'The Pathology of Articular and Spinal Diseases', pp. 74-115. Arnold, London

De Palma, A. F., McKeever, C. D., And Subin, D. K. (1966) Clin. Orthop., 48, 229 (Process of repair of articular cartilage demonstrated by histology and autoradiography with tritiated thymidine)

Elliot, H. C. (1936) Amer. J. Anat., 58, 127 (Studies on articular cartilage. 1. Growth mechanisms)

Fuller, J. A., AND Ghadially, F. N. (1972) Clin. Orthop., 86, 193 (Ultrastructural observations on surgically produced partial-thickness defects in articular cartilage)

Ghadially, F. N., Fuller, J. A., ANd Kirkaldy-Willis, W. H. (1971) Arch. Path., 92, 356 (Ultrastructure of full-thickness defects in articular cartilage)

Hunter, W. (1743) Phil. Trans. 1742-1743, 42, 514 (Of the structure and diseases of articulating cartilages)

Mankin, H. J. (1962a) J. Bone Jt Surg., 44A, 682 (Localization of tritiated thymidine in articular cartilage of rabbits. I. Growth in immature cartilage of rabbits)

- (1962b) Ibid., 44A, 688 (Localization of tritiated thymidine in articular cartilage of rabbits. II. Repair in immature cartilage)

_ (1963) Ibid., 45A, 529 (Localization of tritiated thymidine in articular cartilage of rabbits. III. Mature articular cartilage)

Meachim, G., Ghadially, F. N., and Collins, D. H. (1965) Ann. rheum. Dis., 24, 23 (Regressive changes in the superficial layer of human articular cartilage)

Paget, J. (1853) 'Healing of injuries in various tissues,' in 'Lectures on Surgical Pathology', vol. 1, p. 262. Longmans, London

Redler, I., AND Zimny, M. L. (1970) J. Bone Jt Surg., 52A, 1395 (Scanning electron microscopy of normal and abnormal articular cartilage and synovium)

Walker, P. S., Dowson, D., Longfield, M. D., AND Wright, V. (1968) Ann. rheum. Dis., 27, 512 (Boosted lubrication in synovial joints by fluid entrapment and enrichment)

—, Sikorski, J., Dowson, D., Longfield, M. D., Wright, V., AND Buckley, T. (1969) Ibid., 28, 1 (Behaviour of synovial fluid on surfaces of articular cartilage) 